\title{
$L^{1}$-convergence and hypercontractivity of diffusion semigroups on manifolds
}

\author{
by \\ FENG-Yu WANG (Beijing)
}

\begin{abstract}
Let $P_{t}$ be the Markov semigroup generated by a weighted Laplace operator on a Riemannian manifold, with $\mu$ an invariant probability measure. If the curvature associated with the generator is bounded below, then the exponential convergence of $P_{t}$ in $L^{1}(\mu)$ implies its hypercontractivity. Consequently, under this curvature condition $L^{1}$-convergence is a property stronger than hypercontractivity but weaker than ultracontractivity. Two examples are presented to show that in general, however, $L^{1}$-convergence and hypercontractivity are incomparable.
\end{abstract}

1. Introduction. Let $M$ be a connected, complete, noncompact Riemannian manifold either without boundary or with a convex boundary $\partial M$. Consider the operator $L:=\Delta+Z$, where $Z$ is a $C^{1}$ vector field such that for some $K \geq 0$,

$$
\operatorname{Ric}(X, X)-\left\langle\nabla_{X} Z, X\right\rangle \geq-K|X|^{2}, \quad X \in T M .
$$

Then the (reflecting) $L$-diffusion process is non-explosive (see e.g. [10, Theorem 8.2]). Let $P_{t}$ be the corresponding diffusion semigroup. We assume that $P_{t}$ has a (unique) invariant probability measure $\mu$ (see [3] for a sufficient condition of its existence and uniqueness). In particular, if $Z=\nabla V$ for some $V \in C^{2}(M)$ such that $R:=\int_{M} e^{V(x)} d x<\infty$, where $d x$ stands for the Riemannian volume measure, then $\mu(d x)=R^{-1} e^{V(x)} d x$.

Our main purpose is to compare the $L^{1}$-convergence and hypercontractivity of $P_{t}$. Let us first explain that both properties are stronger than the $L^{2}$-exponential convergence of $P_{t}$.

It is well known that the log-Sobolev inequality implies the Poincaré inequality, and if $P_{t}$ is symmetric then these two inequalities are equivalent, respectively, to the hypercontractivity and $L^{2}$-exponential convergence of $P_{t}$ (see e.g. $[6,8]$ ). Therefore, at least for the symmetric case, the hypercontractivity of $P_{t}$ is stronger than its exponential convergence in $L^{2}(\mu)$. In fact, this implication is also true for the non-symmetric case as soon as (1.1)

2000 Mathematics Subject Classification: 47D07, 60J60.

Supported in part by NNSFC(10025105,10121101), TRAPOYT and the 973-Project. 
holds, since according to [15, Theorem 2.1] (see also [19, Theorem 5.3]) if (1.1) holds then the hypercontractivity of $P_{t}$ is equivalent to the log-Sobolev inequality as well, which in turn implies the Poincaré inequality and hence the $L^{2}$-exponential convergence of $P_{t}$.

On the other hand, suppose that $P_{t}$ converges in $L^{1}(\mu)$, i.e. there is a positive function $\xi$ on $[0, \infty)$ with $\xi(t) \downarrow 0$ as $t \uparrow \infty$ such that

$$
\left\|P_{t}-\mu\right\|_{1 \rightarrow 1} \leq \xi(t), \quad t \geq 0
$$

where $\|\cdot\|_{p \rightarrow q}$ denotes the operator norm from $L^{p}(\mu)$ to $L^{q}(\mu)$, and $\mu(f):=$ $\int_{M} f d \mu$ for $f \in L^{1}(\mu)$. Then, by the semigroup property, $P_{t}$ converges in $L^{1}(\mu)$ exponentially fast, i.e. there exist $c, \lambda>0$ such that

$$
\left\|P_{t}-\mu\right\|_{1 \rightarrow 1} \leq c e^{-\lambda t}, \quad t \geq 0 .
$$

Since $\left\|P_{t}-\mu\right\|_{\infty \rightarrow \infty} \leq 2$ for all $t \geq 0$, by Riesz-Thorin's interpolation theorem (see e.g. [5]) one has

$$
\left\|P_{t}-\mu\right\|_{2 \rightarrow 2} \leq \sqrt{2 c} e^{-\lambda t / 2}, \quad t \geq 0 .
$$

If, in particular, $P_{t}$ is symmetric, then (1.2) implies

$$
\left\|P_{t}-\mu\right\|_{\infty \rightarrow 2}=\left\|P_{t}-\mu\right\|_{2 \rightarrow 1} \leq\left\|P_{t}-\mu\right\|_{1 \rightarrow 1} \leq c e^{-\lambda t}, \quad t \geq 0,
$$

hence according to [14, Theorem 2.3],

$$
\left\|P_{t}-\mu\right\|_{2 \rightarrow 2} \leq e^{-\lambda t}, \quad t \geq 0 .
$$

Therefore, besides hypercontractivity, $L^{1}$-convergence also implies $L^{2}$-exponential convergence.

Our main result says that under condition (1.1), $L^{1}$-exponential convergence is a property between hypercontractivity and ultracontractivity. We refer to $[15,20]$ for explicit sufficient and necessary conditions for these two contractivity properties.

Theorem 1.1. (1) If (1.1) holds, then (1.2) implies the log-Sobolev inequality: there exists $C>0$ such that

$$
\mu\left(f^{2} \log f^{2}\right) \leq C \mu\left(|\nabla f|^{2}\right), \quad \mu\left(f^{2}\right)=1 .
$$

Consequently, the $L^{1}$-convergence of $P_{t}$ implies its hypercontractivity, i.e. for any $t>0$ there exists $p_{t}>2$ such that $\left\|P_{t}\right\|_{2 \rightarrow p_{t}} \leq 1$.

(2) If either (1.1) holds or $P_{t}$ is symmetric, then the ultracontractivity of $P_{t}$ (i.e. $\left\|P_{t}\right\|_{1 \rightarrow \infty}<\infty$ for some $t>0$ ) implies (1.2) for some $c, \lambda>0$.

REMARK 1.2. When $P_{t}$ is symmetric, its $L^{1}$-convergence is equivalent to strong ergodicity:

$$
\lim _{t \rightarrow 0} \sup _{\nu \in \mathcal{P}(M)}\left\|\nu P_{t}-\mu\right\|_{\mathrm{var}}=0
$$

where $\mathcal{P}(M)$ is the set of all probability measures on $M, \nu P_{t} \in \mathcal{P}(M)$ is defined by $\left(\nu P_{t}\right)(A):=\nu\left(P_{t} 1_{A}\right)$ for a measurable set $A$, and $\|\cdot\|_{\text {var }}$ is the 
total variation norm defined by $\|\psi\|_{\text {var }}:=\sup _{A} \psi(A)-\inf _{A} \psi(A)$ for a set function $\psi$. In fact, if $\nu$ is absolutely continuous with respect to $\mu$ then (see e.g. $[4$, Theorem 5.7])

$$
\frac{1}{2}\left\|\nu P_{t}-\mu\right\|_{\mathrm{var}}=\int_{M}\left(P_{t} \frac{d \nu}{d \mu}-1\right)^{+} d \mu=\frac{1}{2} \mu\left(\left|P_{t} \frac{d \nu}{d \mu}-1\right|\right) .
$$

Since $P_{t}(t>0)$ has transition density (see e.g. [7, p. 79]) and since $\mu$ has strictly positive density with respect to the volume measure (see e.g. [3, Theorem 1.1(ii)]), $\nu P_{1}$ is absolutely continuous with respect to $\mu$. Thus, for any $t>1$ one has

$$
\begin{aligned}
\left\|P_{t}-\mu\right\|_{1 \rightarrow 1} & =\sup _{f \geq 0, \mu(f)=1} \mu\left(\left|P_{t} f-1\right|\right) \leq \sup _{\nu \in \mathcal{P}(M)}\left\|\nu P_{t}-\mu\right\|_{\text {var }} \\
& =\sup _{\nu \in \mathcal{P}(M)}\left\|\left(\nu P_{1}\right) P_{t-1}-\mu\right\|_{\text {var }} \\
& =\sup _{\nu \in \mathcal{P}(M)} \mu\left(\left|P_{t-1} \frac{d\left(\nu P_{1}\right)}{d \mu}-1\right|\right) \leq\left\|P_{t-1}-\mu\right\|_{1 \rightarrow 1} .
\end{aligned}
$$

Therefore, in other words, Theorem 1.1(1) means that under (1.1) the strong ergodicity of $P_{t}$ implies the log-Sobolev inequality.

The proof of Theorem 1.1 is given in the next section, while two examples are presented in Section 3 to show that in general $L^{1}$-convergence and hypercontractivity are incomparable.

2. Proof of Theorem 1.1. To prove Theorem 1.1(1), we need the following interpolation theorem due to Peetre [13] (see also [9, Theorem A.1]). In the version below we give an explicit relationship between the relevant constants.

TheOREM 2.1 (Peetre's interpolation theorem). Let $\phi_{0}, \phi_{1}, \phi_{2}$ be three non-negative increasing functions defined on $[0, \infty)$ such that $\phi_{1}=$ $\phi_{0} \sigma\left(\phi_{2} / \phi_{0}\right)$ for a concave function $\sigma$ and $\phi_{i}(2 r) \leq a \phi_{i}(r)$ for some $a>0$ and all $r \geq 0, i=0,1,2$. Let $T$ be a linear operator defined on a space $\mathcal{D}(T) \supset O^{\phi_{i}}:=\left\{f: \mu\left(\phi_{i}(|f|)\right)<\infty\right\}, i=0,1,2$. There exists $c>0$ such that if

$$
\int \phi_{i}(|T f|) d \mu \leq c_{i} \int \phi_{i}(|f|) d \mu, \quad f \in O^{\phi_{i}}, i=0,2
$$

for some $c_{0}, c_{2}>0$, then

$$
\int \phi_{1}(|T f|) d \mu \leq c\left(c_{0} \vee c_{2}\right) \int \phi_{1}(|f|) d \mu, \quad f \in O^{\phi_{1}} .
$$

Proof. For $f \in \mathcal{D}(T)$, define

$$
L(t, f):=\inf _{f=f_{0}+f_{2}}\left\{\mu\left(\phi_{0}\left(\left|f_{0}\right|\right)\right)+t \mu\left(\phi_{2}\left(\left|f_{2}\right|\right)\right)\right\}, \quad t \geq 0 .
$$


By (A.4) in [9], there exist $C \in[1, \infty)$ and a positive measure $\nu$ on $[0, \infty)$ such that for any $f \in \mathcal{D}(T)$,

$$
\frac{1}{C} \int_{0}^{\infty} L(t, f) \nu(d t) \leq \mu\left(\phi_{1}(|f|)\right) \leq C \int_{0}^{\infty} L(t, f) \nu(d t) .
$$

By $(2.1)$,

$$
\begin{aligned}
L(t, T f) & \leq \inf _{f=f_{0}+f_{2}}\left\{\mu\left(\phi_{0}\left(\left|T f_{0}\right|\right)\right)+t \mu\left(\phi_{2}\left(\left|T f_{2}\right|\right)\right)\right\} \\
& \leq \inf _{f=f_{0}+f_{2}}\left\{c_{0} \mu\left(\phi_{0}\left(\left|f_{0}\right|\right)\right)+t c_{2} \mu\left(\phi_{2}\left(\left|f_{2}\right|\right)\right)\right\} \leq\left(c_{0} \vee c_{2}\right) L(t, f) .
\end{aligned}
$$

Combining this with (2.3) we obtain (2.2).

Proof of Theorem 1.1. (1) By (1.1) we have (see $[1,17]$ )

$$
P_{t}\left(f^{2} \log f^{2}\right) \leq \frac{2(\exp [2 K t]-1)}{K} P_{t}|\nabla f|^{2}+\left(P_{t} f^{2}\right) \log \left(P_{t} f^{2}\right), \quad t>0 .
$$

This implies that

$$
\begin{aligned}
& \mu\left(f^{2} \log f^{2}\right) \\
& \quad \leq \frac{2(\exp [2 K t]-1)}{K} \mu\left(|\nabla f|^{2}\right)+\mu\left(\left(P_{t} f^{2}\right) \log P_{t} f^{2}\right), \quad t>0 .
\end{aligned}
$$

To apply Theorem 2.1, let $\phi_{0}(r)=r, \phi_{2}(r)=r^{2}$ and $\phi_{1}(r)=r \log (1+r)$. We have $\sigma(r)=\log (1+r)$, which is concave. Applying Theorem 2.1 to $T:=P_{t}-\mu$ and using (1.2) and (1.3), we obtain

$$
\begin{aligned}
\mu\left(\left|P_{t} f^{2}-1\right| \log (1\right. & \left.\left.+\left|P_{t} f^{2}-1\right|\right)\right) \\
& \leq c_{2} \mu\left(\left|f^{2}-1\right| \log \left(1+\left|f^{2}-1\right|\right)\right) e^{-\lambda_{2} t}, \quad \mu\left(f^{2}\right)=1,
\end{aligned}
$$

for some $c_{2}, \lambda_{2}>0$ and all $t \geq 0$. Therefore, there exists $c_{3}>c_{2}$ such that

$$
\mu\left(P_{t} f^{2} \log P_{t} f^{2}\right) \leq c_{3} e^{-\lambda_{2} t} \mu\left(f^{2} \log f^{2}\right)+c_{3}, \quad t \geq 0, \mu\left(f^{2}\right)=1 .
$$

Combining this with (2.4) for a proper choice of $t>0$, we obtain

$$
\mu\left(f^{2} \log f^{2}\right) \leq A \mu\left(|\nabla f|^{2}\right)+B, \quad \mu\left(f^{2}\right)=1,
$$

for some $A, B>0$. Therefore, to prove the hypercontractivity of $P_{t}$, it suffices to verify the following Poincaré inequality (see e.g. [6, Theorem 6.1.22(ii)]):

$$
\mu\left(f^{2}\right) \leq C \mu\left(|\nabla f|^{2}\right)+\mu(f)^{2}
$$

where $C>0$ is a constant. To this end, we make use of [14, Proposition 3.1], which involves the weak and super Poincaré inequalities. First, since $x \log x \geq R x-e^{R-1}$ for all $x, R \geq 0$, we have (for $\mu\left(f^{2}\right)=1$ )

$$
\begin{aligned}
\mu\left(f^{2} \log f^{2}\right) & =2 \mu\left(f^{2} \log |f|\right) \geq 2 R-2 e^{R-1} \mu(|f|) \\
& \geq 2 R-1-e^{2 R} \mu(|f|)^{2}, \quad R>0 .
\end{aligned}
$$


Combining this with $(2.5)$ we arrive at

$$
\mu\left(f^{2}\right) \leq \frac{A \mu\left(|\nabla f|^{2}\right)+e^{2 R} \mu(|f|)^{2}}{2 R-B-1}, \quad 2 R>B+1 .
$$

Thus, we have the following super Poincaré inequality for some $\beta:(0, \infty) \rightarrow$ $(0, \infty)$ :

$$
\mu\left(f^{2}\right) \leq r \mu\left(|\nabla f|^{2}\right)+\beta(r) \mu(|f|)^{2}, \quad r>0 .
$$

On the other hand, by e.g. [3, Theorem 1.1(ii)] one has $\mu(d x)=e^{V(x)} d x$ for some $V \in C(M)$. Then [14, Theorem 3.1] implies the weak Poincaré inequality, i.e. there exists $\alpha:(0, \infty) \rightarrow(0, \infty)$ such that

$$
\mu\left(f^{2}\right) \leq \alpha(r) \mu\left(|\nabla f|^{2}\right)+r\|f\|_{\infty}^{2}, \quad r>0, \mu(f)=0 .
$$

Therefore, by [14, Proposition 1.3] we obtain (2.6) for some constant $C>0$.

(2) If $P_{t}$ is ultracontractive then (2.5) holds for some constants $A, B>0$ (see e.g. [19, Theorem 5.3]). Thus, as explained above, (2.6) holds and hence $\left\|P_{t}-\mu\right\|_{2 \rightarrow 2} \leq e^{-t / C}, t \geq 0$. Therefore, if $\left\|P_{t_{0}}-\mu\right\|_{1 \rightarrow 2}<\infty$ then for all $t>0$ one has

$$
\left\|P_{t+t_{0}}-\mu\right\|_{1 \rightarrow 1} \leq\left\|P_{t_{0}}-\mu\right\|_{1 \rightarrow 2}\left\|P_{t}-\mu\right\|_{2 \rightarrow 2} \leq e^{-t / C}\left\|P_{t_{0}}-\mu\right\|_{1 \rightarrow 2} .
$$

\section{Incomparability of $L^{1}$-convergence and hypercontractivity.} To show that $L^{1}$-convergence and hypercontractivity are incomparable, let us first recall a result on strong ergodicity which is equivalent to $L^{1}$-convergence for the symmetric case according to Remark 1.2. By Tweedie [16, Theorem 2(iii)] it is well known that for irreducible Markov chains on $\mathbb{Z}_{+}$ strong ergodicity is equivalent to $\sup _{i \in \mathbb{Z}_{+}} E^{i} \tau_{0}<\infty$, where $\tau_{0}$ is the hitting time to 0 and $E^{i}$ is the expectation with respect to the Markov chain starting from $i$. The same has been proved recently by Mao [12] for diffusion processes.

TheOREM 3.1 (Mao [12]). Consider $L:=a(x) d^{2} / d x^{2}+b(x) d / d x$, where $a, b \in C^{1}([0, \infty))$ with $a(x)>0$ for all $x \geq 0$. Let

$$
C(x)=\int_{0}^{x} \frac{b(r)}{a(r)} d r, \quad x \in \mathbb{R} .
$$

Assume that $\int_{0}^{\infty}\left(e^{C(r)} / a(r)\right) d r<\infty$. Then the corresponding reflecting diffusion semigroup $P_{t}$ is strongly ergodic if and only if

$$
\delta:=\int_{0}^{\infty} e^{-C(x)} d x \int_{x}^{\infty} \frac{e^{C(r)}}{a(r)} d r<\infty .
$$

Proof. We include the proof for completeness. Let $\tau_{0}:=\inf \{t \geq 0$ : $\left.x_{t}=0\right\}$, where $x_{t}$ is the reflecting $L$-diffusion process. 
(a) (3.1) is equivalent to $\sup _{x>0} E^{x} \tau_{0}<\infty$. Let

$$
F(x):=\int_{0}^{x} e^{-C(r)} d r \int_{r}^{\infty} \frac{e^{C(s)}}{a(s)} d s, \quad x \geq 0 .
$$

We have $L F(x)=-1$ and hence for $x>0$,

$$
0 \leq E^{x} F\left(x_{\tau_{0} \wedge t}\right)=F(x)-E^{x}\left(\tau_{0} \wedge t\right), \quad t>0 .
$$

Letting $t \rightarrow \infty$ we obtain $E^{x} \tau_{0} \leq F(x)$ and hence (3.1) implies $\sup _{x>0} E^{x} \tau_{0}$ $<\infty$.

Conversely, letting $\tau_{n}:=\inf \left\{t \geq 0: x_{t} \geq n\right\}$ we have

$$
\begin{aligned}
F(x) & =E^{x} \tau_{0} \wedge \tau_{n}+E^{x} F\left(\tau_{0} \wedge \tau_{n}\right) \\
& \leq F(n) P^{x}\left(\tau_{n}<\tau_{0}\right)+E^{x} \tau_{0}, \quad n>x .
\end{aligned}
$$

Since for $G(x):=\int_{0}^{x} e^{-C(r)} d r$ one has $L G=0$, it follows that

$$
F(x)=E^{x} G\left(x_{\tau_{0} \wedge \tau_{n}}\right)=G(n) P^{x}\left(\tau_{n}<\tau_{0}\right), \quad n>x .
$$

Combining this with (3.2) we arrive at

$$
F(x) \leq E^{x} \tau_{0}+\frac{F(n) G(x)}{G(n)}, \quad n>x .
$$

This implies that $F(\infty)<\infty$ provided $\sup _{x>0} E^{x} \tau_{0}<\infty$. Indeed, if $F(\infty)$ $=\infty$, then since $\int_{0}^{\infty}\left(e^{C(r)} / a(r)\right) d r<\infty$, we have $G(\infty)=\infty$ and $F(n) / G(n)$ $\rightarrow 0$ as $n \rightarrow \infty$. Thus, by letting $n \rightarrow \infty$, we see from (3.3) that $F(x) \leq E^{x} \tau_{0}$ for all $x>0$ and hence $\sup _{x>0} E^{x} \tau_{0}=\infty$.

(b) Strong ergodicity implies $\sup _{x>0} E^{x} \tau_{0}<\infty$. If $P_{t}$ is strongly ergodic, then there exists $t>0$ such that $\inf _{x>1} P^{x}\left(x_{t} \leq 1\right) \geq \frac{1}{2} \mu([0,1])=: c>0$, where $\mu$ is the invariant probability measure. Thus,

$$
\begin{aligned}
P^{x}\left(\tau_{1}>2 t\right) & \leq P^{x}\left(x_{t}>1, x_{2 t}>1\right)=E^{x} 1_{\left\{x_{t}>1\right\}} P^{x_{t}}\left(x_{t}>1\right) \\
& \leq(1-c)^{2}, \quad x>1 .
\end{aligned}
$$

Similarly, we have

$$
P^{x}\left(\tau_{1}>n t\right) \leq(1-c)^{n}, \quad x>1 .
$$

Therefore,

$$
E^{x} \tau_{1}=\int_{0}^{\infty} P^{x}\left(\tau_{1}>s\right) d s \leq \delta<\infty
$$

for some $\delta>0$ and all $x>1$.

On the other hand, by the proof in (a) we see that

$$
E^{x} \tau_{1}=\int_{1}^{x} e^{-C(r)} d r \int_{r}^{\infty} \frac{e^{C(s)}}{a(s)} d s, \quad x>1 .
$$


Then $\int_{0}^{\infty}\left(e^{C(s)} / a(s)\right) d s<\infty$ and

$$
\sup _{x>0} E^{x} \tau_{0}=\sup _{x>1} E^{x} \tau_{0}=\sup _{x>1} E^{x} \tau_{1}+\int_{0}^{1} e^{-C(r)} d r \int_{r}^{\infty} \frac{e^{C(s)}}{a(s)} d s<\infty .
$$

(c) $\sup _{x>0} E^{x} \tau_{0}<\infty$ implies strong ergodicity. For any $x>y \geq 0$, let $\left(x_{t}, y_{t}\right)$ be a coupling of the reflecting $L$-diffusion process with $x_{0}=x$, $y_{0}=y$. We have

$$
T:=\inf \left\{t \geq 0: x_{t}=y_{t}\right\} \leq \tau_{0}:=\inf \left\{t \geq 0: x_{t}=0\right\} .
$$

As usual we let $x_{t}=y_{t}$ for $t>T$ so that for any measurable set $A$ we have $\left|\delta_{x} P_{t}(A)-\delta_{y} P_{t}(A)\right| \leq E^{x, y}\left|1_{A}\left(x_{t}\right)-1_{A}\left(y_{t}\right)\right| \leq P^{x, y}\left(x_{t} \neq y_{t}\right) \leq P^{x, y}(T>t)$.

Therefore,

$$
\begin{aligned}
\sup _{x>y \geq 0}\left\|\delta_{x} P_{t}-\delta_{y} P_{t}\right\|_{\mathrm{var}} & \leq 2 \sup _{x>y \geq 0} P^{x, y}(T>t) \leq 2 \sup _{x>0} P^{x}\left(\tau_{0}>t\right) \\
& \leq \frac{\sup _{x>0} E^{x} \tau_{0}}{t}
\end{aligned}
$$

which goes to zero as $t \rightarrow \infty$. This means that $P_{t}$ is strongly ergodic.

ExAmple 3.1. Consider the Ornstein-Uhlenbeck operator $L:=d^{2} / d x^{2}-$ $x d / d x$ on $[0, \infty)$. It is well known that the semigroup $P_{t}$ of the reflecting $L$-diffusion process is hypercontractive. But according to Theorem 3.1, $P_{t}$ is not strongly ergodic since (3.1) does not hold. Therefore, $P_{t}$ does not converge in the $L^{1}$-norm by Remark 1.2 .

ExAMPLE 3.2. Let $M=[0, \infty)$ and consider $L:=d^{2} / d x^{2}+b(x) d / d x$, where

$$
b(x):=-\frac{\gamma^{\prime}(x)}{\gamma(x)}-\frac{1}{\gamma(x)}, \quad x \geq 0,
$$

with $\gamma$ constructed as follows. For any $n \geq 1$, let $\phi_{n} \in C^{\infty}[0, \infty)$ be nonnegative such that $\left.\phi_{n}\right|_{\left[n, n+e^{-n}\right]^{\mathrm{c}}}=0$ and

$$
\left.\phi_{n}\right|_{\left[n+e^{-n} / 4, n+3 e^{-n} / 4\right]}=\max \phi_{n}=e^{n}(1+n)^{-2} .
$$

Set $\gamma(r)=(1+r)^{-2}+\sum_{n \geq 1} \phi_{n}(r), r \geq 0$. Then $P_{t}$ is strongly ergodic and hence $L^{1}$-convergent but not hypercontractive.

Proof. We have

$$
C(x):=\int_{0}^{x} b(r) d r=-\log \gamma(x)-\int_{0}^{x} \frac{d r}{\gamma(r)}, \quad x \geq 0 .
$$

Then

$$
\exp [-C(x)]=\gamma(x) \exp \left[\int_{0}^{x} \frac{d r}{\gamma(r)}\right], \quad \exp [C(x)]=-\frac{d}{d x} \exp \left[-\int_{0}^{x} \frac{d r}{\gamma(r)}\right] .
$$


Therefore, $\int_{0}^{\infty} e^{C(x)} d x=1$ and

$$
\int_{0}^{\infty} e^{-C(x)} d x \int_{x}^{\infty} e^{C(y)} d y=\int_{0}^{\infty} \gamma(x) d x \leq \int_{0}^{\infty} \frac{d r}{1+r^{2}}+\sum_{n=1}^{\infty} \frac{1}{1+n^{2}}<\infty .
$$

Thus, (3.1) holds and hence $P_{t}$ is strongly ergodic.

On the other hand, we use [2, Theorem 1.1] to disprove the log-Sobolev inequality. Observe that

$$
\begin{aligned}
\frac{1}{3}(1+x)^{3} & \geq \int_{0}^{x} \frac{d r}{\gamma(r)} \geq \frac{1}{3}(1+x)^{3}-\sum_{n \geq 1} \int_{n}^{n+e^{-n}}(1+r)^{2} d r \\
& \geq \frac{1}{3}(1+x)^{3}-\sum_{n \geq 1}(2+n)^{2} e^{-n}=: \frac{1}{3}(1+x)^{3}-c_{1}
\end{aligned}
$$

Then for $\mu([0, x]):=\int_{0}^{x} e^{C(r)} d r$,

$$
\begin{aligned}
\int_{0}^{\infty} e^{-C(x)} \mu([0, x]) d x & =\int_{0}^{\infty} \gamma(x)\left(\exp \left[\int_{0}^{x} \frac{d r}{\gamma(r)}\right]-1\right) d x \\
& \geq e^{-c_{1}} \int_{0}^{\infty} \frac{e^{(1+x)^{3} / 3}}{(1+x)^{2}} d x-\int_{0}^{\infty} \gamma(x) d x=\infty
\end{aligned}
$$

since $\int_{0}^{\infty} \gamma(x) d x<\infty$. Moreover,

$$
\begin{aligned}
I(n) & :=\left(\int_{0}^{n+e^{-n}} e^{-C(y)} d y\right)\left(\int_{n+e^{-n}}^{\infty} e^{C(y)} d y\right)\left(\log \frac{1}{\int_{n+e^{-n}}^{\infty} e^{C(y)} d y}\right) \\
& =\left(\int_{0}^{n+e^{-n}} \gamma(y) \exp \left[\int_{0}^{y} \frac{d r}{\gamma(r)}\right] d y\right) \exp \left[-\int_{0}^{n+e^{-n}} \frac{d r}{\gamma(r)}\right]\left(\int_{0}^{n+e^{-n}} \frac{d r}{\gamma(r)}\right) \\
& \geq c_{2}\left(\int_{n+e^{-n} / 4}^{n+3 e^{-n} / 4} \frac{e^{n}}{(1+n)^{2}} \exp \left[(1+y)^{3} / 3\right] d y\right) e^{-(1+n)^{3} / 3}(1+n)^{3} \\
& \geq c_{3}(1+n)-c_{4}
\end{aligned}
$$

for some $c_{2}, c_{3}, c_{4}>0$. Thus $\lim _{n \rightarrow \infty} I(n)=\infty$. Therefore, according to $[2$, Theorem 1.1] the log-Sobolev inequality does not hold; see also [11] for a more general result.

Acknowledgements. The author would like to thank Professors MuFa Chen, Yong-Hua Mao and Liming Wu for useful conversations. He also thanks the referee for helpful comments.

\section{References}

[1] D. Bakry and M. Ledoux, Lévy-Gromov's isoperimetric inequality for an infinite dimensional diffusion generator, Invent. Math. 123 (1996), 259-281. 
[2] S. G. Bobkov and F. Götze, Exponential integrability and transportation cost related to logarithmic Sobolev inequalities, J. Funct. Anal. 163 (1999), 1-28.

[3] V. I. Bogachev, M. Röckner and F. Y. Wang, Elliptic equations for invariant measures on finite and infinite dimensional manifolds, J. Math. Pures Appl. 80 (2001), $177-221$.

[4] M. F. Chen, From Markov Chains to Non-Equilibrium Particle Systems, World Sci., Singapore, 1992.

[5] E. B. Davies, Heat Kernels and Spectral Theory, Cambridge Univ. Press, 1989.

[6] J. D. Deuschel and D. W. Stroock, Large Deviations, Pure Appl. Math. 137, Academic Press, San Diego, 1989.

[7] K. D. Elworthy, Geometric aspects of diffusions on manifolds, in: Lecture Notes in Math. 1362, 277-425, Springer, 1988, 277-425.

[8] L. Gross, Logarithmic Sobolev inequalities and contractivity properties of semigroups, in: Lecture Notes in Math. 1563, Springer, 1993, 54-88.

[9] J. Gustavsson and J. Peetre, Interpolation of Orlicz spaces, Studia Math. 60 (1977), $33-59$.

[10] X. M. Li, Strong p-completeness of stochastic differential equations and the existence of smooth flows on noncompact manifolds, Probab. Theory Related Fields 100 (1994), 485-511.

[11] Y. H. Mao, Logarithmic Sobolev inequalities for birth-death processes and diffusion processes on the line, Chinese J. Appl. Probab. Statist. 18 (2002), 94-100.

[12] - Strong ergodicity for Markov processes by coupling methods, J. Appl. Probab. 39 (2002), 839-852.

[13] J. Peetre, A new approach in interpolation spaces, Studia Math. 34 (1970), 23-42.

[14] M. Röckner and F. Y. Wang, Weak Poincaré inequalities and $L^{2}$-convergence rates of Markov semigroups, J. Funct. Anal. 185 (2001), 564-603.

[15] -, -, Supercontractivity and ultracontractivity for (non-symmetric) diffusion semigroups on manifolds, Forum Math. 15 (2003), 893-921.

[16] R. L. Tweedie, Criteria for ergodicity, exponential ergodicity and strong ergodicity of Markov processes, J. Appl. Probab. 18 (1981), 122-130.

[17] F. Y. Wang, On estimation of the logarithmic Sobolev constant and gradient estimates of heat semigroups, Probab. Theory Related Fields 108 (1997), 87-101.

[18] —, Functional inequalities for empty essential spectrum, J. Funct. Anal. 170 (2000), 219-245.

[19] —, Functional inequalities, semigroup properties and spectrum estimates, Infin. Dimens. Anal. Quantum Probab. Relat. Top. 3 (2000), 263-295.

[20] - , Logarithmic Sobolev inequalities: conditions and counterexamples, J. Operator Theory 46 (2001), 183-197.

Department of Mathematics

Beijing Normal University

Beijing 100875, P.R. China

E-mail: wangfy@bnu.edu.cn

Received August 12, 2002

Revised version December 1, 2003 